\title{
Diacronie
}

Studi di Storia Contemporanea

$\mathrm{N}^{\circ} 20,4 \mid 2014$

II diritto militante

\section{Il quarto governo Fanfani e la crisi di Cuba del}

\section{2}

Una chiave di volta per l'apertura a sinistra

\section{Matteo Anastasi}

\section{(2) OpenEdition \\ Journals}

Edizione digitale

URL: http://journals.openedition.org/diacronie/1748

DOI: 10.4000/diacronie. 1748

ISSN: 2038-0925

Editore

Association culturelle Diacronie

Notizia bibliografica digitale

Matteo Anastasi, « II quarto governo Fanfani e la crisi di Cuba del 1962 », Diacronie [Online], № 20,

4 | 2014, documento 11, Messo online il 01 décembre 2014, consultato il 19 avril 2019. URL : http:// journals.openedition.org/diacronie/1748; DOI : 10.4000/diacronie.1748 


\section{Diacronie}

N. 20 | 4|2014 Il diritto militante

\section{1/}

\section{Il quarto governo Fanfani e la crisi di Cuba del 1962}

\section{Una chiave di volta per l'apertura a sinistra}

Matteo ANASTASI *

Il presente saggio è incentrato sull'analisi del ruolo giocato dall'Italia durante uno dei momenti di maggiore attrito della guerra fredda: la crisi dei missili di Cuba. La scelta di questo tema deriva dalla volontà di esaminare un frangente della storia contemporanea particolarmente battuto, quale la tensione dell'ottobre 1962, alla luce dell'azione italiana nella crisi, quest'ultimo aspetto, invece, relativamente poco studiato. Obiettivo del lavoro è dimostrare da un lato l'impegno profuso da Roma per favorire una soluzione pacifica della contesa, dall'altro la profonda influenza che gli eventi cubani, e più in generale, l'acuirsi delle tensioni sovietico-statunitensi, ebbero sulle dinamiche politiche italiane.

\section{Introduzione}

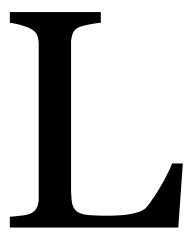

'intento principale di questo saggio è mostrare l’importanza della crisi di Cuba sulle dinamiche politiche interne dell'Italia dei primi anni Sessanta. Le vicende dell'autunno 1962 andavano, infatti, a coincidere con un momento di profondo cambiamento del panorama politico italiano, culminato nella formazione del quarto gabinetto di Amintore Fanfani, esecutivo che aprì ai socialisti rompendo la precedente tradizione legata al "centrismo", elemento dominante sin dalla fine degli anni Quaranta. Il coinvolgimento italiano nella crisi cubana derivava dalla presenza nella penisola di missili Jupiter, dislocati fra Puglia e Basilicata fin dal 1960. Essi, puntati verso est su richiesta della Casa Bianca, furono, insieme ai missili collocati 
in territorio turco, al centro del braccio di ferro ingaggiato da Kennedy e Chruščëv nell'ottobre 1962. La presenza dei missili all'interno del paese fece si che «il caso italiano fosse [...] sempre presente [nelle trattative fra sovietici e statunitensi], direttamente o indirettamente, sin da quando venne ricercata e trovata la via di un compromesso pacifico»1. Soluzione alla crisi che sarà raggiunta anche grazie all'intenso lavoro diplomatico intrapreso dal governo di Roma e in particolare da Fanfani, che si attiverà a più riprese per convincere Kennedy a rinunciare allo scontro frontale.

\title{
2. La questione dei missili Jupiter
}

A partire dagli anni Cinquanta, il possesso di armi nucleari sempre più potenti determinò, fra Stati Uniti e Unione Sovietica, un costante confronto tecnologico sul piano della realizzazione di missili a lunga gittata. Tale competizione si inasprì quando $\mathrm{i}$ sovietici, poco dopo aver testato con successo un $\mathrm{Icbm}^{2}$, superarono con i loro esperimenti quelli di Washington, riuscendo a portare in orbita per la prima volta un satellite, lo Sputnik, il 4 ottobre del 1957.

I due test effettuati da Mosca ebbero fortissime ripercussioni negli Stati Uniti poiché dimostravano che gli avversari sarebbero stati in grado di raggiungere e colpire con missili a testata atomica il suolo americano, fino a quel frangente immune da pericoli diretti di questo tipo3. Allo stesso modo, in Europa, i paesi alleati svilupparono forti preoccupazioni per la loro sicurezza, aspettandosi dalla Casa Bianca risposte strategiche valide a contrastare la crescente potenza sovietica. Come ha ricordato David Schwartz:

\begin{abstract}
When viewed from the perspective of Washington, the Europeans seemed to have conflicting concerns. On the one hand, they worried that the United States would not execute strategic retaliation in response to aggression against Western Europe; for Washington to do so would be suicidal. On the other hand, they worried about
\end{abstract}

\footnotetext{
${ }^{1}$ DI NOLFO, Ennio, La guerra fredda e l'Italia, Firenze, Polistampa, 2010, p. 421.

2 L'Icbm, acronimo dell'espressione inglese Intercontinental Ballistic Missile, è un missile balistico per il trasporto a lungo raggio di ordigni nucleari. Esso si distingue da altri missili balistici, come gli Irbm (Intermediate Range Ballistic Missile) e gli Srbm (Short Range Ballistic Missile), per la sua ampia gittata, superiore a cinquemila $\mathrm{km}$. Cfr. CARTER, Ashton, SCHWARTZ, David, Ballistic Missile Defense, Washington, The Brookings Institution, 1984, pp. 122-153. Il primo Icbm della storia, l'R-7 Semërka, fu lanciato in orbita dai sovietici il 21 agosto 1957. Cfr. GADDIS, John Lewis, La guerra fredda: rivelazioni e riflessioni, Milano, Mondadori, 2007 , p. 471.

3 Cfr. NASH, Philip, The other missiles of october: Eisenhower, Kennedy and the Jupiters 19571963, London, North Carolina University Press, 1997, p. 13.
} 
what would happen if the United States did execute a strategic retaliation in response to aggression against Europe, because that could provoke Soviet retaliation against entire Nato alliance; the Europeans would have no influence on a decision that would reduce their society to rubble4.

La sicurezza europea contro un eventuale attacco comunista dipendeva dunque totalmente dal sostegno degli Stati Uniti e dal loro potenziale atomico, per cui gli Stati membri dell'Alleanza atlantica guardarono da subito con grande interesse a quali sarebbero stati i provvedimenti che Washington avrebbe preso dopo il lancio dello Sputnik. La reazione politica immediata dell'amministrazione Eisenhower fu di rinforzare militarmente il blocco occidentale con i mezzi di cui disponeva, e cioè con missili di raggio intermedio che solo da basi europee avrebbero potuto raggiungere e colpire quelle sovietiche. Nel dicembre del 1957, durante una riunione del Consiglio atlantico a Parigi, il presidente Eisenhower informò gli alleati dei nuovi e necessari programmi di difesa, proponendo l'installazione di missili balistici sul territorio di quei partner che avessero accettato il progetto 5 . Il fatto che per la prima volta, da quando l'Alleanza aveva avuto origine, alla riunione fossero presenti in rappresentanza dei paesi membri gli stessi capi di Stato, dimostrava come vi fosse grande tensione intorno agli eventi di quei mesi6.

Fra tutti gli alleati, ancor prima dell'inverno 1957, la Gran Bretagna era stata la prima a essere coinvolta nei progetti di Washington. Infatti, in seguito all'intesa raggiunta con il governo statunitense dal primo ministro Harold MacMillan durante la conferenza delle Bermuda del marzo 1957 , Londra aveva già posto le premesse per raggiungere un accordo con gli Stati Uniti analogo a quello che Eisenhower avrebbe

\footnotetext{
4 SCHWARTZ, David, NATO's Nuclear Dilemmas, Washington, The Brookings Institution, 1983, p. 35 .

5 «Durante il Consiglio atlantico di Parigi del 16-19 dicembre 1957, gli Stati Uniti, per superare il cosiddetto missile gap, proposero la dislocazione di missili del tipo Jupiter e Thor in alcuni paesi europei, fra cui l'Italia». FERRARIS, Luigi Vittorio, Manuale della politica estera italiana, Bari, Laterza, 1996, p. 113.

${ }^{6}$ Cfr. NUTI, Leopoldo, La sfida nucleare. La politica estera italiana e le armi atomiche 19451991, Bologna, Il Mulino, 2007, p. 139.

7 Il summit alle Bermuda fu organizzato congiuntamente da MacMillan ed Eisenhower per dimostrare pubblicamente il permanere di buoni rapporti fra Londra e Washington, dopo che gli eventi di Suez dell'anno precedente avevano fatto sorgere delle frizioni fra le due amministrazioni. In questa circostanza, dopo aver ricordato cordialmente il servizio militare prestato fianco a fianco in nord Africa durante la seconda guerra mondiale, i due leader discussero della questione mediorientale e raggiunsero un accordo in base al quale MacMillan si impegnava a ospitare sul suolo britannico sessanta missili statunitensi Irbm del tipo Thor. Cfr. MAMMARELLA, Giuseppe, Europa-Stati Uniti. Un'alleanza difficile (1945-1973), Firenze, Vallecchi, 1973, p. 198.
} 
successivamente proposto agli altri membri dell'Alleanza ${ }^{8}$. Gli inglesi avevano alcune importanti ragioni per accettare gli Irbm nel loro territorio. Anzitutto i Thor sarebbero stati operativi prima dei Blue Streak, «che erano dei missili a medio raggio alla cui realizzazione la Gran Bretagna aveva rivolto i suoi sforzi fino a quel momento»9, e il dispiegamento dei missili statunitensi avrebbe consentito anche di acquisire informazioni interessanti riguardo tecnologie più avanzate, permettendo in tal modo un'accelerazione nella fabbricazione dei Blue Streak. Inoltre, ospitare i missili americani avrebbe permesso un notevole risparmio di fondi da dedicare al potenziamento delle armi convenzionali; infine, con questo accordo, la special relationship fra i due paesi sarebbe uscita particolarmente rinsaldata, come era nelle intenzioni del governo britannico dopo gli eventi di Suez. Anche gli Stati Uniti, dal canto loro, vedevano nel dispiegamento dei Thor in Gran Bretagna, non solo un significativo rafforzamento sul piano strategico, ma anche altri vantaggi, fra cui la possibilità che gli inglesi lasciassero da parte il loro progetto sui missili Irbm e dedicassero tutta la loro attenzione al potenziamento delle armi convenzionali ${ }^{10}$.

Il fatto che dal dicembre 1957 la proposta di detenere vettori nucleari fosse stata estesa anche agli altri alleati, ai quali fino a quel momento la Casa Bianca non aveva concesso di partecipare attivamente alla gestione diretta delle armi atomiche, fa immaginare lo stato di assoluta urgenza in cui l'amministrazione Eisenhower si trovò per porre rimedio alla sensazione di crescente inferiorità nei confronti dei sovietici. Bisogna comunque considerare che
nell'ambito della strategia del new look, seguita dall'amministrazione di Washington fin dai primi mesi del 1953, il fatto di ampliare la quantità di armi nucleari possedute al fine di scoraggiare [...] ogni iniziativa minacciosa della controparte, era considerato di normale attuazione, così come del resto le armi

\footnotetext{
8 «In early 1957, Eisenhower formally accepted the principle of providing the British with Irbm. He did not insist or even suggest at the time that these missiles would be subject to Nato control. The agreement with Britain simply called for missiles to be "made available by the United States for use by British forces" and said nothing at all about Nato. It was clear that Eisenhower intended to treat the British generously. In late 1957, he wanted to "take the British into the fold on the basis of mutual confidence", and called for a full exchange of information". TRACHTENBERG, Marc, A Construed Peace. The Making of the European Settlement 19451963, Princeton, Princeton University Press, 1999, pp. 207-208.

9 SORRENTI, Deborah, L'Italia nella guerra fredda. La storia dei missili Jupiter, Roma, Edizioni Associate, 2003, p. 12.

10 «The Thors might convince the British to abandon Blue Streak. This would indirectly strengthen Western defense by preventing wasteful duplication and preserving British funds for conventional forces, while it would also head off what might become a fully independent British missile capability». NASH, Philip, The other missiles of october: Eisenhower, Kennedy and the Jupiters 1957-1963, cit., pp. 10-11.
} 
nucleari erano valutate al pari di quelle convenzionali. Di conseguenza il tipo di strategia che si pensò di attuare faceva parte comunque di un disegno già studiato $\mathrm{e}$ predeterminato senza che potesse apparire come una misura straordinaria ${ }^{11}$.

Tuttavia, la condivisione del proprio arsenale nucleare con gli alleati poneva vari problemi ai dirigenti della Casa Bianca, dal momento che il McMahon Act ${ }^{12}$, approvato dal Congresso degli Stati Uniti nel 1946, proibiva espressamente di fornire informazioni o armi nucleari a paesi terzi. Eisenhower doveva perciò trovare una formula che consentisse di venire incontro alle richieste degli europei senza violare apertamente la legge ${ }^{13}$. In merito alla politica nucleare da seguire, il Congresso e il presidente avevano, infatti, opinioni divergenti, poiché il numero uno della Casa Bianca riteneva eccessivamente restrittiva la legge che regolava la condivisione e il possesso delle armi atomiche statunitensi con gli alleati ${ }^{14}$.

Il problema del controllo delle testate atomiche era inoltre strettamente connesso alla questione della Germania. Mosca si opponeva all'eventualità che la Germania federale ricevesse dai suoi alleati armi atomiche di cui potesse condividerne l'utilizzo, poiché ciò avrebbe rappresentato una minaccia quanto mai temibile in caso di guerra, almeno fino a quando il governo di Bonn non avesse riconosciuto come legittima l'esistenza della Germania orientale. I sovietici avrebbero preferito che la Germania divenisse un territorio neutrale, "perché ciò avrebbe rappresentato la logica premessa per la definitiva stabilizzazione delle frontiere dell'Europa centrale, e quindi non avrebbero accettato che la Repubblica federale tedesca potesse avere un arsenale atomico sotto il suo diretto controllo» ${ }^{15}$. Contrariamente il presidente Eisenhower era convinto che la Germania ovest, facendo parte dell'Alleanza atlantica, non potesse

${ }^{11}$ SORRENTI, Deborah, L'Italia nella guerra fredda. La storia dei missili Jupiter, cit., p. 13.

12 Il McMahon Act (noto anche come Atomic Energy Act) fu firmato dal presidente Harry Truman nell'agosto 1946 ed entrò in vigore nel gennaio dell'anno successivo. Ispirato dal senatore democratico del Connecticut Brien McMahon, tale provvedimento affermò il monopolio di Washington nella gestione e nel controllo della tecnologia nucleare sviluppata congiuntamente da Stati Uniti, Gran Bretagna e Canada durante il secondo conflitto mondiale. Cfr. LEVI, Edward Hirsch, Memories about the McMahon Act, Chicago, University of Chicago Press, 1984, pp. 1-3.

${ }_{13}$ Cfr. NASH, Philip, The other missiles of october: Eisenhower, Kennedy and the Jupiters 1957-1963, cit., p. 12.

${ }^{14}$ In un discorso ufficiale dell'ottobre 1957 Eisenhower enunciò chiaramente le sue perplessità riguardo il McMahon Act: "The Atomic Energy Act had been a great mistake. The policy it mandated seemed to run counter to common sense', he told De Gaulle in September 1959. 'The law', he told the French leader a few months later, was 'somewhat absurd'. [...] The president took exactly the same line in meetings with United States officials. In May 1959, for example, he complained about being handcuffed by the 'senseless limitations' Congress had placed on the administration». TRACHTENBERG, Marc, A Construed Peace. The Making of the European Settlement 1945-1963, cit., p. 197.

${ }^{15}$ SORRENTI, Deborah, L'Italia nella guerra fredda. La storia dei missili Jupiter, cit., p. 14. 
essere discriminata rispetto agli altri Stati membri e che quindi dovesse avere accesso all'armamento atomico degli occidentali. I sovietici insistevano per affermare le proprie ragioni facendo leva su quello che in Europa era un punto debole, e cioè puntando sul problema di Berlino e sulla questione del riconoscimento della Germania orientale da parte dei paesi occidentali. Come messo in luce dallo storico statunitense Marc Trachtenberg, che ha dedicato uno studio approfondito a questi problemi:

There were two basic ways of dealing with the issue of control of a Nato, and eventually a European, nuclear force. In both cases the problems were enormous. The first alternative was for this force to be organized in the same way as the rest of Nato on a national basis, under ultimate national control, but with plans worked out within the Nato structure, and with a unified command becoming fully effective in the event of war. Planning, and especially targeting, could be done primarily on an alliance-wide basis, although there was no reason why arrangements for various fallback contingencies could not also be worked out on a national basis. [...] The second and more basic problem with a Nato or European nuclear force organized on a national basis was that it meant Germany finger on the nuclear trigger. A German state with a nuclear capability of its own would no longer be locked into a purely defensive policy; and if it looked like Germany was developing a nuclear force under national control, the Soviets might be tempted to act before it was too late ${ }^{16}$.

Rispetto ai rapporti che gli Stati Uniti avevano con il governo inglese, quelli con gli altri alleati determinavano quindi una situazione più complessa, per cui il programma presentato al Consiglio atlantico del 1957, a parte l'immediata risposta entusiastica della Turchia ${ }^{17}$, non trovò facilmente altre offerte di collaborazione. Si può affermare che, dinanzi alla proposta di Eisenhower, i paesi alleati si divisero in tre gruppi, individuabili in base alle loro reazioni: quelli favorevoli all'attuazione del programma, e cioè Francia, Olanda e Turchia, altri sei paesi che assunsero un atteggiamento più contrastato, ovvero Belgio, Repubblica federale tedesca, Grecia, Italia, Lussemburgo e Portogallo, e gli ultimi due, Danimarca e Norvegia, che furono nettamente contrari ${ }^{18}$. Infine, ad accettare la possibilità di ospitare le armi offerte dagli Stati Uniti furono soltanto Italia e Turchia, in quanto gli altri paesi presentarono problemi politici interni

16 TRACHTENBER, Marc, A Construed Peace. The Making of the European Settlement 19451963, cit., pp. 201-203.

${ }_{17}$ Cfr. NASH, Philip, The other missiles of october: Eisenhower, Kennedy and the Jupiters 19571963, cit., p. 18.

18 Cfr. Ibidem. 
o di altra natura che non consentirono la conclusione dell'accordo. Per esempio, benché la Francia si trovasse in cima alla lista dei possibili ospitanti e avesse espresso apertamente il proprio interesse per il programma degli Irbm già prima dell'annuncio che Eisenhower aveva fatto alla Nato, non si riuscì a raggiungere con essa alcuna intesa. A Parigi si ambiva a realizzare un progetto nucleare autonomo, e perciò si considerava assai preziosa la collaborazione con gli Stati Uniti. Tuttavia i negoziati sui missili Jupiter si arenarono sul punto riguardante il controllo delle testate, che i francesi volevano sotto la loro diretta responsabilità, senza dipendere né dall'Alleanza né dal suo "comandante" in Europa. Obiettivo del Quai d'Orsay era di occupare una posizione privilegiata nei rapporti con Washington analoga a quella detenuta dalla Gran Bretagna ${ }^{19}$. Il dialogo franco-statunitense si protrasse a lungo (passando in mezzo alle vicende politiche che in Francia portarono alla caduta della Quarta Repubblica e al ritorno al governo di De Gaulle) ma, vista l'intransigenza transalpina, il negoziato fu definitivamente abbandonato nell'autunno 1958. I militari statunitensi, del resto, già da alcuni mesi avevano iniziato le consultazioni con i rappresentanti degli altri paesi, assegnando una priorità secondaria a quelle con i francesi ${ }^{20}$.

Nella Germania federale la possibilità di ospitare gli $\mathrm{Irbm}$ si unì a un più ampio dibattito sulla questione delle armi nucleari. Sebbene Adenauer, condividendo il parere di Washington, ritenesse opportuno lo schieramento di quei missili, sia l'opinione pubblica tedesca sia le forti perplessità dei militari, condizionarono l'andamento dei negoziati, finché lo stesso Supreme Allied Commander Europe (Saceur) concluse che non era possibile raggiungere alcun accordo ${ }^{21}$.

La Turchia era stata invece l'unico paese ad aver immediatamente dimostrato la propria disponibilità verso i progetti statunitensi. Il governo di Ankara, infatti, considerava di grande rilevanza l'occasione di possedere armi atomiche al fine di conseguire il prestigio internazionale che le sarebbe derivato da una stretta collaborazione con la Casa Bianca.

In Italia, intanto, le elezioni del maggio 1958 erano state vinte dalla Democrazia cristiana con il 42,4\% dei voti ${ }^{22}$. Tale esito elettorale rese possibile la formazione di un esecutivo moderato (costituito dal partito cattolico insieme ai socialdemocratici e con l'appoggio esterno dei repubblicani) guidato da Amintore Fanfani, il quale ambiva a

19 Cfr. SCHWARTZ, David, NATO's Nuclear Dilemmas, cit., pp. 69-70.

${ }^{20}$ Cfr. NASH, Philip, The other missiles of october: Eisenhower, Kennedy and the Jupiters 1957-1963, cit., pp. 53-56.

${ }^{21}$ Ibidem.

${ }_{22}$ Cfr. NUTI, Leopoldo, Gli Stati Uniti e l'apertura a sinistra: importanza e limiti della presenza americana in Italia, Bari, Laterza, 1999, p. 128. 
realizzare, oltre a una serie di riforme sociali e economiche, anche una politica estera "neo-atlantica", in conformità della quale l'Italia avrebbe dovuto svolgere un ruolo più attivo ed autonomo nelle relazioni internazionali. Uno dei principali obiettivi del nuovo corso di Fanfani era aumentare l'influenza diplomatica di Roma nel Medio Oriente, attribuendole un ruolo di mediazione nei rapporti fra quest'area e gli Stati Uniti, come del resto la crisi di Suez aveva fatto sperare con la disfatta di Francia e Gran Bretagna. Per muoversi in tale direzione, tuttavia, era necessario innanzitutto rafforzare i rapporti con l'alleato principale e quindi manifestare a Washington una fedeltà politica e un appoggio diplomatico indiscussi ${ }^{23}$.

Se si accetta questa interpretazione, sembra plausibile ipotizzare che la disponibilità a ospitare i missili Jupiter con testate atomiche, non fosse quindi per l'Italia una scelta sostenuta semplicemente da motivi di sicurezza collettiva, ma bensì il risultato dell'applicazione di una politica che rispondeva soprattutto a una logica di tipo nazionale. Nell'accettare la proposta statunitense per il dispiegamento di quelle armi, l'Italia si metteva in una condizione di assoluto prestigio anche perché, essendo divenuta di conseguenza un bersaglio per eventuali attacchi sovietici, avrebbe potuto avere dalla Nato una più cospicua assistenza militare, senza così doversi sobbarcare un aumento delle spese riguardanti le armi convenzionali ${ }^{24}$. Ciò si sposava perfettamente con la volontà statunitense di trovare alleati disponibili all'attuazione del programma ${ }^{25}$.

Un altro punto da mettere a fuoco, per meglio comprendere il senso della decisione italiana, sono le caratteristiche del tipo di arma in discussione, che la rendeva di per sé poco interessante agli occhi degli alleati: i missili Jupiter erano in posizione fissa, facilmente individuabili e difficili da nascondere al nemico. La nazione destinata a ospitarli assumeva perciò il rischio di divenire il primo e immediato obiettivo di un attacco, al quale forse non avrebbe potuto rispondere in tempo utile ${ }^{26}$. In sostanza si trattava di armi che «avevano [...] un valore politico molto più alto di quello militare. Esse costituivano il simbolo di una reazione immediata a un eventuale attacco dei

${ }^{23}$ Cfr. Ibidem, pp. 130-155. A due mesi dalla nomina a capo del governo, il 27 luglio 1958, Fanfani rilasciò un'intervista al Washington Post nella quale dichiarò a chiare lettere la fedeltà italiana nei confronti della Casa Bianca. Lo statista aretino affermò: «La più completa e operante solidarietà atlantica è, oggi come ieri, la stella polare della politica estera italiana». WOLLEMBORG, Leo, Stelle, strisce e tricolore. Trent'anni di vicende politiche fra Roma e Washington, Milano, Mondadori, 1983, p. 65.

24 Cfr. NUTI, Leopoldo, «Dall'operazione Deep Rock all'operazione Pot Pie: una storia documentata dei missili SM 78 Jupiter in Italia», in Storia delle Relazioni Internazionali, 1, 1996-1997, p. 107.

25 Ibidem, pp. 146-147.

${ }^{26}$ Cfr. CORALLUZZO, Valter, La politica estera dell'Italia repubblicana 1946-1992. Modello di analisi e studio di casi, Milano, Franco Angeli, 2000, p. 116. 
sovietici, della costante presenza di Washington a difesa degli europei, del prestigio che conferivano al paese che le deteneva» 27 .

Gli accordi fra i due governi furono conclusi attraverso uno scambio di note che, differentemente da un vero e proprio trattato, avrebbe permesso di aggirare le eventuali difficoltà politiche che la sinistra avrebbe potuto frapporre in Parlamento al momento della ratifica ${ }^{28}$. Fu la necessità di evitare questa possibile reazione negativa a convincere Fanfani a procedere con la dovuta cautela, invitando gli Stati Uniti a condurre a termine la missione con il massimo riserbo, facendola apparire, non come un incremento dell'arsenale atomico già presente in Italia, ma come un'attività militare di normale attuazione ${ }^{29}$.

Durante i negoziati, che si svolsero fra l'estate del 1958 e il marzo del 1959, il governo di Washington ebbe qualche esitazione a concedere a quello italiano margini decisionali sull'uso delle armi in questione. Preoccupazione americana era quella di evitare equivoci che avrebbero potuto consentire un'interpretazione allargata degli accordi anche riguardo l'utilizzo degli altri armamenti nucleari statunitensi, già presenti nel territorio italiano, ma sotto l'assoluta responsabilità della Casa Bianca. Come messo in luce da Leopoldo Nuti,

il dipartimento di Stato suggeriva di evitare che nelle proposte presentate formalmente agli italiani ci fosse qualche riferimento, da parte americana, alla formula sull'uso congiunto dei missili: una clausola del genere, infatti, poteva pregiudicare eventuali intese su altre armi nucleari che in quel momento si trovavano in Italia e che erano controllate dagli Stati Uniti; inoltre sarebbe stato

\footnotetext{
${ }^{27}$ SORRENTI, Deborah, L'Italia nella guerra fredda. La storia dei missili Jupiter, cit., p. 18. 28 «[...] Fanfani aveva chiesto che l'accordo fosse perfezionato nella forma di uno scambio di note, per evitarne il passaggio parlamentare e le prevedibili polemiche delle sinistre. [...] Gli Stati Uniti acconsentirono a tale richiesta e l'intesa fu siglata il 26 marzo 1959 con uno scambio di note tra il ministro degli Esteri Pella e l'ambasciatore americano a Roma Zellerbach. Se fu possibile aggirare il voto delle Camere con l'artificio dello scambio di note non si evitarono però le polemiche. La reazione in Parlamento fu vivace e trovò concordi i deputati comunisti e socialisti, seppur con numerosi distinguo, nello stigmatizzare l'accordo che portava l'Italia, secondo la valutazione dei parlamentari dell'opposizione di sinistra, ad abdicare alla sovranità nazionale e ad esporsi al rischio di distruzione atomica. [...] Successivamente anche le dichiarazioni di Pella a Washington in occasione della riunione convocata per il decimo anniversario della Nato suscitarono una vivace polemica per lo spirito bellicoso che le sinistre attribuivano al governo. Il ministro aveva affermato: 'Se mia figlia dovesse correre il rischio di vivere in un mondo comunista, io come padre scelgo per la mia bambina piuttosto il rischio della bomba atomica'». MARTELLI, Evelina, L'altro atlantismo: Fanfani e la politica estera italiana (1958-1963), Milano, Guerrini e Associati, 2008, pp. 114-115. L'accordo raggiunto fra Roma e Washington «poneva le basi per la dislocazione sul territorio italiano di 30 missili a media gittata [...] Jupiter, che sarebbero divenuti operativi sin dal 1960, prima dell'ascesa alla presidenza di Kennedy». DI NOLFO, Ennio, La guerra fredda e l'Italia, cit., p. 424.

29 Cfr. NUTI, Leopoldo, «Dall'operazione Deep Rock all'operazione Pot Pie: una storia documentata dei missili SM 78 Jupiter in Italia», cit., pp. 97-101.
} 
desiderabile mantenere il più a lungo possibile il pieno controllo de facto degli Stati Uniti e del Saceur in merito all'impiego degli $\operatorname{Irbm}^{30}$.

Il problema venne comunque sollevato da parte italiana e si risolse con l'adozione di una formula di compromesso che prevedeva la possibilità di un uso congiunto dei missili, previo ordine del Saceur e in base agli impegni reciproci assunti. Per quanto concerneva invece il lancio vero e proprio, esso sarebbe stato effettuato da una squadra costituita da tecnici italiani comandati dal loro ufficiale, «il quale avrebbe tenuto sempre appesa al collo, per motivi di sicurezza e prontezza, la chiave che avrebbe permesso l'avvio del conto alla rovescia. Un'altra chiave sarebbe stata tenuta da un ufficiale americano e sarebbe servita invece per effettuare l'ultimo passaggio del count down»31. Le testate nucleari, di cui tutti i missili erano forniti, sarebbero dovute rimanere in ogni caso sotto la custodia delle autorità statunitensi, collocate a parte e non montate stabilmente sui vettori. Il punto centrale degli accordi restava però per gli italiani la questione economica, poiché il governo precisò immediatamente che il proprio sostegno allo schieramento dei Jupiter non corrispondeva a un'analoga disponibilità ad assumersi l'intero costo dell'operazione ${ }^{22}$. Roma chiese perciò che una quota degli investimenti fosse sostenuta da Washington e così avvenne, poiché le spese intraprese da parte italiana si limitarono sostanzialmente alla fornitura dei terreni e alla copertura dei costi per la realizzazione delle strutture33. Il generale Lauris Norstad, comandante supremo delle forze Nato in Europa, in seguito a numerose consultazioni con i militari italiani, decise che il sito strategicamente migliore su cui collocare le rampe missilistiche fosse il sud della penisola, in particolare le zone della Puglia e della Basilicata. Fra queste due regioni vennero individuate dieci località 34 , a bassa densità abitativa e abbastanza lontane dai centri più popolosi, nelle quali sarebbero sorte le postazioni di lancio, ognuna ospitante tre missili.

30 Ibidem, p. 102.

${ }^{31}$ SORRENTI, Deborah, L'Italia nella guerra fredda. La storia dei missili Jupiter, cit., pp. 1920.

${ }^{32}$ Cfr. TRACHTENBERG, Marc, A Construed Peace. The Making of the European Settlement 1945-1963, cit., pp. 146-200.

33 In particolare durante le trattative si stabilì che il governo americano avrebbe contribuito all'operazione con una somma totale di oltre dodici milioni di dollari, più una quota ulteriore destinata agli imprevisti. A ciò si sarebbe aggiunta anche la fornitura di materiali e servizi per lo stesso ammontare, mentre il governo italiano si impegnò a finanziare la costruzione delle strutture delle basi missilistiche, il loro funzionamento e la loro manutenzione per una spesa annua di circa nove milioni di dollari. Cfr. NUTI, Leopoldo, «Dall'operazione Deep Rock all'operazione Pot Pie: una storia documentata dei missili SM 78 Jupiter in Italia», cit., pp. 111112.

34 Le dieci località erano: Acquaviva delle Fonti, Altamura (Castel Sabini e Santeramo), Gioia del Colle e Gravina in Puglia, Laterza, Mottola e Spinazzola in Puglia; Irsina e Matera in Basilicata. Cfr. SORRENTI, Deborah, L'Italia nella guerra fredda. La storia dei missili Jupiter, cit., p. 10. 
Le basi rimasero attive per tre anni, dal gennaio 1960 all'aprile 1963, quando vennero smantellate in seguito agli accordi intervenuti fra Stati Uniti ed Unione Sovietica dopo la crisi di Cuba. Il ritiro dei Jupiter italiani fu una conseguenza diretta dell'intesa informale fra Kennedy e Chruščëv, in base alla quale Mosca avrebbe rinunciato alle sue basi missilistiche sul territorio caraibico in cambio della rimozione dei vettori nucleari collocati in Turchia (le rampe turche, infatti, più vicine di quelle italiane al territorio sovietico, costituivano per il Cremlino una minaccia particolarmente concreta). I missili italiani vennero in seguito sostituiti, tra la primavera e l'estate del 1963, con sistemi più moderni ed efficaci installati su sommergibili, i cosiddetti missili Polaris. Essi, collocati su basi mobili e forniti di propellente solido, divenivano un obiettivo molto più difficile da colpire e quindi anche più sicuro. Inoltre, a differenza dei Jupiter, i Polaris non erano soggetti al controllo congiunto dei due governi, ma erano posti esclusivamente sotto la custodia degli Stati Uniti. Il presidente Kennedy, infatti, aveva gradualmente mutato la strategia nucleare di Washington, fino ad attuare una politica molto diversa da quella voluta dal suo predecessore Eisenhower: fra gli scopi della nuova teoria della flexible response ${ }^{35}$, vi era anche quello di tenere maggiormente sotto controllo l'arsenale atomico in Europa, cercando di incoraggiare gli alleati a partecipare sempre più attivamente alle spese convenzionali per la propria difesa. Ciò provocò una certa apprensione fra i governi europei che iniziarono a temere che la Casa Bianca cominciasse a propendere per un disimpegno nella difesa del continente, soprattutto per quanto potesse concernere l'aspetto specificamente finanziario ${ }^{36}$. In realtà, dal punto di vista di Kennedy, l'opzione di Eisenhower, secondo il quale era auspicabile che anche la Germania federale potesse avere accesso all'arsenale nucleare dell'Alleanza (sviluppando così un potenziale che facesse da contrappeso alla potenza sovietica in Europa), permettendo agli statunitensi di ridurre il loro impegno nella difesa europea, era assolutamente irrealizzabile. Il nuovo presidente propendeva per il mantenimento dello status quo, aumentando il controllo degli Stati Uniti sulle armi atomiche collocate nei territori degli alleati europei, e mirando a raggiungere un dialogo sempre più efficace con i sovietici37.

35 La strategia della flexible response prevedeva risposte proporzionali, da parte della Casa Bianca, in base alla gravità delle situazioni da affrontare. Si trattava di un'inversione di tendenza rispetto alla massive retaliation, sostenuta in precedenza dall'amministrazione Eisenhower, e richiedente risposte militari rapide e decise dinanzi ad ogni minaccia nemica. Cfr. DAALDER, Ivo, The Nature and Practice of Flexible Response. Nato Strategy and Theater Nuclear Forces since 1967, New York, Columbia University Press, 1991, pp. 4-16.

${ }^{36}$ Cfr. SORRENTI, Deborah, L'Italia nella guerra fredda. La storia dei missili Jupiter, cit., p. 21.

37 «Kennedy hoped the two major powers could find some way to live with each other in peace [...] A European force that included a German contingent was out of the question, because it 


\section{Il ruolo di mediazione italiano}

Le intenzioni moderate di Kennedy, a un anno e mezzo dalla sua ascesa alla Casa Bianca, furono messe a dura prova dalla crisi di Cuba. La gestione dell'emergenza ebbe carattere prettamente bipolare e l'Europa, che era all'origine della tensione e ne era la posta in gioco, non fu coinvolta dagli Stati Uniti nelle decisioni che il presidente varò appoggiandosi all'Executive Committee of the National Security Council (Excomm), il comitato di esperti costituito da Kennedy per fronteggiare la crisi $3^{8}$. Fin dalle prime discussioni del comitato esecutivo di Washington, l'Italia assunse un ruolo centrale, poiché ospitava basi missilistiche americane ed era uno dei possibili bersagli della rappresaglia sovietica. Secondo le decisioni stabilite in tale consesso, Roma doveva essere informata preventivamente dell'eventuale decisione statunitense di attaccare Cuba39. Oltre a Gran Bretagna, Francia e Repubblica federale tedesca, Kennedy inviò pertanto una lettera al presidente del Consiglio italiano. In essa chiedeva di garantire il sostegno agli Stati Uniti in sede Onu e si diceva certo che Fanfani gli avrebbe comunicato le sue valutazioni rispetto al collegamento di tale iniziativa con altre in Europa. Infine, ribadì l'estrema importanza di un lavoro a stretto contatto e in piena armonia, nonché il proprio impegno a informare l'alleato degli ulteriori sviluppi. Fanfani diede notizia della missiva al presidente della Repubblica Segni e al ministro degli Esteri Piccioni e disse all'ambasciatore statunitense a Roma Reinhardt di temere

meant in the final analysis a German finger on the nuclear trigger, and a force built up from British and French contingents alone would scarcely solve Germany's security problem, or lead the Germans to accept a permanent non-nuclear status. [...] The assumption was that the development of a German nuclear force would be a source of instability both in itself and because of the likely Soviet reaction. [...] And that general policy implied that the U.S. government would have to tighten control over American nuclear weapons with the Nato forces in Europe. The deployment of American strategic missiles on European soil would also have to be opposed, because these weapons might fall too easily into the hand of host country forces». TRACHTENBERG, Marc, A Construed Peace. The Making of the European Settlement 19451963, cit., pp. 283-285.

${ }^{8}$ Del comitato, ufficializzato il 16 ottobre, facevano parte il numero due della Casa Bianca Lyndon Johnson, il segretario di Stato Dean Rusk, il segretario della Difesa Robert McNamara, il direttore della Cia John McCone, Robert Kennedy e i più stretti consiglieri del presidente. Cfr. SMITH, Thomas, Encyclopedia of the Central Intelligence Agency, New York, Facts on File, 2003, p. 90.

39 Cfr. NUTI, Leopoldo, L'Italie et les missiles Jupiter, in VAÏSSE, Maurice (a cura di), L'Europe et la crise de Cuba, Parigi, Armand Colin, 1993, p. 140. In particolare l'Excomm stabilì, in caso di via libera all'offensiva sull'isola caraibica, di avvertire preventivamente MacMillan, Adenauer, De Gaulle e, solo nell'imminenza dell'attacco, Fanfani e i turchi. La decisione di informare Palazzo Chigi solo all'ultimo momento era dettata dalla volontà di impedire che Roma tentasse di opporsi all'eventuale offensiva statunitense. Cfr. MARTELLI, Evelina, L'altro atlantismo: Fanfani e la politica estera italiana (1958-1963), cit., p. 390. 
che i sovietici volessero far scattare una trappola che avrebbe avuto ripercussioni a Berlino e altrove. Il 23 ottobre Fanfani rispose a Kennedy con una lunga lettera in cui dichiarava la comprensione e la solidarietà dell'Italia per il difficile frangente che gli Stati Uniti stavano attraversando:

L'Italia comprende e sente quali vive preoccupazioni per il mantenimento della pace nel mar dei Caraibi, nell'emisfero occidentale e in ogni altra parte del mondo susciti il concentrarsi di armi nucleari offensive nell'isola di Cuba. Però mancherei ad ogni elementare dover di amicizia [...] se non dicessi che le misure da Lei decise possono far correre a tutti gravissimi pericoli. Perciò sono stati apprezzati: il ricorso degli Stati Uniti all'Onu, che noi appoggeremo; il Suo appello diretto al primo ministro Chruščëv; la Sua calorosa riconferma di essere disposto a qualsiasi esame in qualsiasi sede di tutti gli altri gravi problemi che oggi turbano il malfermo equilibrio su cui poggia l'instabile pace del mondo. Come Ella da tempo ben sa, signor presidente, fermamente credo che, poggiando sulla sicura volontà di pace degli Stati Uniti, utilizzando in primo luogo la sede dell'Onu e giungendo ad una discussione esauriente ed anche diretta tra Lei e il signor Chruščëv, è possibile, con un costruttivo esame globale di tutti i problemi oggi aperti, far passare il mondo dall'attuale gravissima tensione all'inizio di una nuova fase di tranquillità. Occorre giungere con coraggio e determinazione a un esame globale di tutti i problemi esistenti. E questo esame deve essere il primo obiettivo di coloro che oggi hanno la massima responsabilità nella condotta del mondo, e cioè i capi degli Stati Uniti e dell'Unione Sovietica. Ritengo che altri paesi e altri uomini potranno o sapranno affiancare e sostenere una tanto ardita, ma indilazionabile operazione. L'Italia e i suoi governanti certamente l'appoggerebbero con la stessa convinzione con la quale la auspicano [...]. Che Iddio ed il consiglio di sinceri amici della Sua persona e del Suo paese l'assistano, signor presidente, in questo momento decisivo per tutti. Tra gli amici che Le sono vicini, auspicando il Suo pieno successo nelle opere di pace e di difesa della libertà, si conferma cordialmente il Suo devotissimo A. Fanfani40.

Lo stesso 23 ottobre Fanfani ricevette una lettera di MacMillan. Il premier britannico gli proponeva di mantenere strettissimi contatti e lo informava di aver assicurato a Kennedy il pieno appoggio del Regno Unito all'Onu. Fanfani gli rispose lo stesso giorno dicendosi favorevole a un continuo aggiornamento reciproco sull'evoluzione della crisi. Si dichiarò molto preoccupato per l'estrema gravità della

40 Lettera di Amintore Fanfani a John F. Kennedy, Roma, 23 ottobre 1962, citata in GENTILONI SILVERI, Umberto, Fanfani visto da Washington, in GIOVAGNOLI, Agostino, TOSI, Luciano (a cura di), Amintore Fanfani e la politica estera italiana: atti del convegno di studi (Roma, 3-4 febbraio 2009), Venezia, Marsilio, 2010, pp. 113-114. 
situazione e, non essendo l'Italia in quel momento nel Consiglio di sicurezza delle Nazioni Unite, affermò di poter solo auspicare che in tale sede si trovasse una soluzione pacifica.

Fanfani intervenne poi in Parlamento, mentre Piccioni si trovava a Bruxelles per la riunione dei ministri degli Esteri dei Sei, da dove rientrò il giorno successivo ${ }^{41}$. Il presidente del Consiglio dichiarò alla Camera il pieno appoggio del governo agli Stati Uniti, ma «fece anche appello al dialogo per la rimozione delle cause che avevano spinto Washington al blocco e al mantenimento del contatto fra Washington e Mosca»42. Fanfani proseguì:

proprio nel momento in cui più intensi si svolgono i dialoghi per il disarmo e la tregua nucleare, appaiono contraddittori gli atti rivolti a creare basi dove non esistono, ad accrescere preoccupazioni ove già ne esistono, ad aggravare il già troppo precario equilibrio, dal cui mantenimento, in attesa di auspicabili ragionevoli e leali accordi, dipende ancora la malferma pace del mondo. L'Italia giudica positivo il fatto che il governo degli Stati Uniti abbia chiesto all'Onu di decidere un intervento che sotto controllo internazionale accerti ed elimini le cause dell'allarme stesso43.

Tornato a Roma, Piccioni convocò gli ambasciatori statunitense e sovietico, poi inviò Carlo Russo, sottosegretario agli Esteri, a New York per seguire le trattative e dirigere la delegazione italiana alle Nazioni Unite con l'incarico di favorire l'azione del segretario generale U Thant.

Il 26 ottobre, giorno in cui Kennedy ricevette la lettera privata di Chruščëv che gli proponeva la smobilitazione in cambio dell'assicurazione di non invadere Cuba, Russo incontrò l'ambasciatore americano presso l'Onu Adlai Stevenson, che lo ringraziò per le parole di appoggio agli Stati Uniti pronunciate in Parlamento da Fanfani e gli chiese un giudizio sull'ipotesi di uno scambio tra il ritiro dei missili nell'isola caraibica e di quelli in basi europee, specie se superati ${ }^{44}$. Russo interpretò questa richiesta come puramente

\footnotetext{
${ }^{41}$ Come ha ricordato Evelina Martelli, «la concomitanza della crisi di Cuba fece sì che nella riunione fossero discusse principalmente le questioni internazionali ed in particolare le possibili ripercussioni della crisi nei Carabi su Berlino» mentre, invece, «non furono fatti progressi nelle trattative europee». MARTELLI, Evelina, L'altro atlantismo: Fanfani e la politica estera italiana (1958-1963), cit. p. 391.

42 Ibidem.

43 VILLANI, Angela, L'Italia e l'ONU negli anni della coesistenza competitiva (1955-1968), Padova, Cedam, 2007, p. 166.

44 Cfr. MARTELLI, Evelina, L'altro atlantismo: Fanfani e la politica estera italiana (19581963), cit., p. 392.
} 
esplorativa e rispose che sarebbe stato preferibile negoziare la smobilitazione delle postazioni missilistiche europee nel quadro della conclusione delle trattative sul disarmo, ma non opporre ostacoli di principio. L'Italia seguì l'evoluzione della crisi e cercò di incoraggiare i negoziati principalmente dal foro delle Nazioni Unite, dove U Thant stava trattando per evitare l'escalation nucleare. Il segretario generale dell'Onu aveva rivolto il 24 ottobre un appello a Chruščëv, chiedendo la sospensione dell'invio di armi, e a Kennedy, domandando la dilazione del blocco navale. Nei giorni successivi proseguì la febbrile attività del palazzo di vetro per una mediazione e un'individuazione delle misure di controllo e di assicurazione reciproca fra Stati Uniti e Unione Sovietica.

Kennedy non fece ricorso alle Nazioni Unite e rispose ufficialmente al messaggio di Chruščëv del 26 ottobre dichiarando la disponibilità a non invadere l’isola e la generica condiscendenza a lavorare per un accordo più generale riguardante altri armamenti. Simultaneamente Robert Kennedy, ministro degli Esteri di Washington, incontrò l'ambasciatore sovietico Dobrynin al ministero di Giustizia accordandosi sul compromesso sostanziale che prevedeva lo smantellamento dei missili in Turchia, e accessoriamente in Italia, in cambio della smobilitazione da Cuba45. L'amministrazione statunitense conosceva la posizione del governo italiano che, diversamente da quello turco, era favorevole al ritiro dei Jupiter, auspicandone la sostituzione con mezzi più adeguati e sicuri46 ${ }^{6}$. Il ministro della Difesa, Andreotti, aveva ripetutamente ribadito tale

In realtà quella avanzata da Stevenson era, per i dirigenti della Casa Bianca, più che una semplice ipotesi. La necessità di rimuovere le basi statunitensi in Europa in cambio di un'azione analoga dei sovietici a Cuba, era emersa come unica soluzione alla crisi fin dalla metà di ottobre: «Sin dal 19 ottobre McNamara sostenne la necessità di un compromesso basato sulla rinuncia alle basi turche ed italiane, spingendosi persino ad ipotizzare una rinuncia alla base americana di Guantanamo, nell'isola di Cuba. Una posizione, questa, condivisa anche da Adlai Stevenson [...]. A tale ipotesi si opponevano risolutamente soltanto i militari ed alcuni "falchi" della diplomazia americana [tutti presenti all'interno dell'Excomm], come Paul Nitzte [assistente del segretario alla Difesa per gli affari internazionali di sicurezza], Maxwell Taylor [membro dello Stato Maggiore statunitense] e Douglas Dillon [segretario del Tesoro]. Lo stesso presidente Kennedy condivideva questa posizione». DI NOLFO, Ennio, La guerra fredda e l'Italia, cit., p. 428.

45 «Uno dei momenti centrali per l'evoluzione della crisi fu l'impegno segreto, preso da Robert F. Kennedy il 27 ottobre [...] con il nuovo ambasciatore sovietico a Washington Anatoly Dobrynin, di smantellare le basi di missili Jupiter situate sul territorio della Turchia e, in subordine, anche quelli posti in Italia, entro un termine ragionevole, dopo il ritiro dei missili sovietici da Cuba». DI NOLFO, Ennio, L'Italie et la crise de Cuba en 1962, in VAÏSSE, Maurice (sous la dir. de), L'Europe et la crise de Cuba, cit., p. 109.

46 La disponibilità italiana era ben nota all'amministrazione statunitense. A tal proposito l'ambasciatore americano alla Nato, Thomas K. Finletter, in una lettera indirizzata a Rusk, scriveva: «L'ipotesi di smobilitare i missili da Turchia ed Italia presenta problemi specialmente in relazione ad Ankara, che non sembra interessata all'obsolescenza dei mezzi ed al fatto che sono facili bersagli quanto piuttosto al fatto che essi rappresentano la garanzia americana contro l'invasione dell'Unione Sovietica. Sembra pertanto preferibile, essendo Cuba fuori dall'area Nato, proporre la smobilitazione di basi esterne a tale area. Ci sembra che invece gli italiani siano più propensi a smantellare i missili Jupiter, se si trova un'adeguata sostituzione». 
disponibilità e, proprio alla vigilia dei fatti di Cuba, lo aveva confermato a McNamara47. L'esecutivo di Roma sosteneva, infatti, con forza una soluzione pacifica alla crisi ed era a tal fine impegnato sul fronte delle trattative all'Onu. Sia nella lettera a Kennedy che nell'intervento in Parlamento Fanfani aveva appoggiato la soluzione negoziale ed espresso la condiscendenza all'apertura di colloqui su tutte le questioni che erano suscettibili di turbare la pace internazionale.

La posizione assunta dal presidente del Consiglio e dal suo governo erano, inoltre, congeniali anche per l'effettiva convergenza della posizione governativa con quella dei socialisti. Nel Psi la corrente degli autonomisti, guidata da Nenni, si schierò su posizioni pacifiste, chiedendo un serrato dialogo tra Stati Uniti e Unione Sovietica e il ricorso alla mediazione dell'Onu ${ }^{48}$. Si trattava di una posizione coerente con quanto era emerso in occasione dell'ultimo congresso socialista, il XXXIV, tenutosi a Milano fra il 15 e il 19 marzo del 1961. In questa circostanza, la contrapposizione fra autonomisti nenniani - la cui linea sarebbe prevalsa con duecentosettantamila voti favorevoli e duecentocinquemila contrari49 - e sinistre interne si era manifestata duramente. In politica interna, Nenni aveva accelerato la marcia verso l'area democratica, staccandosi in maniera netta dai comunisti, tanto sul piano ideologico quanto su quello politico. Viceversa, le sinistre interne, guidate da Basso e Vecchietti, «pur facendo dichiarazioni di adesione alla politica di autonomia del partito, tentavano di fatto di ricondurre il Psi su posizioni di solidarietà e in definitiva di subordinazione nei confronti dei comunisti ${ }^{50}$. In politica estera, gli autonomisti si presentarono come difensori del

KEEFER, Edward, Cuban Missile Crisis and Aftermath, Washington, United States Government Printing Office, 1997, p. 202. Analogamente l'ambasciata statunitense a Roma comunicava, il 26 ottobre, che la rimozione dei missili dall'Italia «sarebbe probabilmente fattibile», essendo sufficiente che il governo di Washington consultasse preventivamente quello italiano. Cfr. Ibidem, p. 215.

47 Cfr. KEEFER, Edward, Cuban Missile Crisis and Aftermath, cit., p. 205. «Andreotti incontrò McNamara alla vigilia della crisi e gli disse che gli italiani sarebbero stati lieti di rinunciare ai vecchi missili, se gli americani lo avessero voluto». DI NOLFO, Ennio, La guerra fredda e l'Italia, cit., p. 428.

48 «Il governo di centro-sinistra italiano accettò la quarantena [decretata da Kennedy alle navi sovietiche in rotta verso Cuba] con una certa reticenza. Durante la crisi, soprattutto all'interno del palazzo di vetro, esso fece il possibile per migliorare le relazioni con i sovietici e per spingere Kennedy verso un negoziato con Chruščëv. Quando, il 27 ottobre, l'attacco americano parve imminente, il primo ministro democristiano, Amintore Fanfani, chiese a Kennedy di rinviare la scadenza dell'ultimatum e si dichiarò favorevole a un compromesso concernente i Jupiter dislocati in Turchia. Il Psi, dal quale dipendeva il fragile equilibrio della coalizione, aveva condannato la quarantena e senza dubbio aveva incoraggiato negoziati che mettessero fine alla crisi». BERNSTEIN, Barton Bernstein, «The Cuban Missile Crisis: Trading the Jupiter in Turkey?», in Political Science Quarterly, 95, 1/1980, p. 101.

49 Cfr. PARTITO SOCIALISTA ITALIANO, $34^{\circ}$ Congresso nazionale, Milano, Ed. Avanti!, 1961, p. 9.

$5^{\circ}$ D'AURIA, Elio, Gli anni della «difficile alternativa». Storia della politica italiana 1956-1976, Napoli, ESI, 1983, p. 108. 
Patto Atlantico, ritenuto fattore oramai ineliminabile per un paese come l'Italia, geograficamente e culturalmente occidentale. Tale posizione si scontrò accesamente con quella delle sinistre interne, filo-comuniste e ostili alla Nato51. A complicare ulteriormente il quadro intervenne, circa un anno più tardi, la posizione dell'autonomista Riccardo Lombardi che, l'11 gennaio 1962 presso il Comitato centrale del Psi, sottolineò come i socialisti accettassero la Nato solo in chiave difensiva. Tale posizione era già stata chiaramente espressa da Nenni. Lombardi la riprese, precisando, tuttavia, che l'espressione «difensiva» era da intendersi esclusivamente come monito a non favorire un riarmo tedesco52.

Ad ogni modo, durante la crisi di Cuba, le perplessità italiane circa il ricorso di Washington alla quarantena e il costante riferimento alle Nazioni Unite quale foro competente per il negoziato si conciliavano, di fatto, con le manovre politiche interne. Tuttavia è necessario sottolineare come esse rispondessero altresì ad alcuni basilari obiettivi di politica estera, svincolati dalle considerazioni relative alle alleanze domestiche. L'Italia fu, infatti, costantemente una tenace sostenitrice del ruolo dell'Onu per attutire la tensione est-ovest e per gestire le crisi bipolari.

L'adesione totale dell'Italia alle decisioni statunitensi circa la risoluzione della crisi e la corrispondenza delle posizioni dei due esecutivi portò un "atlantista" intransigente quale Sergio Fenoaltea, ambasciatore a Washington, a descrivere così i rapporti tra i due Stati: «L'amministrazione Kennedy ha una grande fiducia verso il primo ministro Fanfani e considera l'Italia, soprattutto con Fanfani alla sua guida, come uno dei suoi alleati più fedeli e più importanti»53. Fenoaltea telegrafò a Roma dopo il discorso del presidente del Consiglio al Parlamento, comunicando che esso era stato ben accolto alla Casa Bianca ed era stato interpretato come la manifestazione di un appoggio totale a Kennedy.

Più sfumato fu l'apprezzamento nei confronti italiani del dipartimento di Stato americano, per i distinguo che avevano caratterizzato la posizione di Roma rispetto a Washington nel corso della crisi ${ }^{54}$. Qui si giudicava che i motivi dell'appoggio incondizionato fossero da ricercarsi nella necessità di rafforzare politicamente l'asse di centro-sinistra, nell'orgoglio nazionale e nella maggiore prosperità del paese. Tali

${ }^{51}$ Cfr. VOULGARIS, Yannis, L'Italia del centro-sinistra (1960-1968), Roma, Carocci, 1998, pp. 111-116.

$5^{2}$ Cfr. MARTELLI, Evelina, L'altro atlantismo: Fanfani e la politica estera italiana (19581963), cit., pp. 340-341.

53 DI NOLFO, Ennio, La guerra fredda e l'Italia, cit., p. 431.

54 Il Dipartimento di Stato di Washington, infatti, si era espresso contrariamente all'intervento dell'Onu, richiesto invece a più riprese da Fanfani. Cfr. VILLANI, Angela, L'Italia e l'ONU negli anni della coesistenza competitiva (1955-1968), cit., p. 166. 
fattori, anche se nel complesso positivi, avrebbero comportato in futuro un appoggio meno scontato e totale dell'Italia alla politica statunitense55.

Diverso fu invece il giudizio che ne diedero alcuni organi di stampa italiani, che considerarono l'intervento governativo come manifestazione di una pericolosa tendenza al neutralismo e all'indebolimento dell'Alleanza atlantica56. Nel dibattito alla Camera sul bilancio degli Esteri, che si svolse dal 26 al 30 ottobre, si delinearono posizioni molto variegate e al governo pervennero critiche da settori della destra, anche interni alla Dc, che accusarono l'esecutivo di aver sostenuto troppo timidamente gli Stati Uniti57. Il deputato del Pri Randolfo Pacciardi parlò a tal proposito di

55 Cfr. NUTI, Leopoldo, Gli Stati Uniti e l'apertura a sinistra: importanza e limiti della presenza americana in Italia, cit., p. 551. Agli occhi del dipartimento di Stato e della diplomazia statunitense (in particolare dell'ambasciata a Roma), l'azione del governo italiano era stata pesantemente condizionata dall'atteggiamento di Nenni che, non volendo perdere terreno rispetto ai comunisti in vista delle prossime elezioni politiche, aveva aderito ad una linea dichiaratamente filo-statunitense. Tuttavia, secondo i funzionari di Washington, tale posizione, non essendo altro che il frutto di scelte di convenienza, sarebbe potuta venir meno in breve tempo e con grande facilità. Cfr. DI NOLFO, Ennio, La guerra fredda e l'Italia, cit., p. 432. La diffidenza del dipartimento di Stato nei confronti dei socialisti si evince da una missiva inviata alla fine dell'ottobre 1962 da Arthur Schlesinger, come si è detto fra i maggiori fautori dell'apertura a sinistra in Italia, a McGeorge Bundy, National Security Advisor del presidente Kennedy: «Da quindici mesi [scriveva Schlesinger] la Casa Bianca si sforza di convincere il dipartimento di Stato che un atteggiamento di simpatia verso i socialisti di Nenni favorirebbe gli interessi degli Stati Uniti e delle democrazie occidentali. [...] Durante tutto questo periodo quasi tutti gli eventi hanno dimostrato la fondatezza della nostra opinione secondo la quale i socialisti di Nenni hanno definitivamente rotto i ponti con i comunisti e sono risoluti a far aderire il loro partito alla causa della democrazia. [...] Ciò nonostante, durante questo periodo il Dipartimento di Stato ha ostacolato ogni possibilità d'intesa tale da accelerare l'inserimento dei socialisti nel campo democratico». DI SCALA, Spencer, Renewing Italian Socialism. Nenni to Craxi, New York, Oxford University Press, 1988, p. 93.

${ }^{6}$ Fra gli altri, «Il Giornale d'Italia», avrebbe commentato in data 18 novembre: «La linea della nostra politica estera inquieta i gruppi più coscienti [...] della Democrazia cristiana [...]. Non è esagerato affermare che alcuni settori della Democrazia cristiana, suggestionati e spinti dal neutralismo del Psi, stanno divenendo, non neutralisti, ma "pacifisti". Dal pacifismo a un atlantismo emascolato e inerte non c'è che un passo: più esattamente, non v'è alcun passo, sono la stessa cosa». Citato in MARTELLI, Evelina, L'altro atlantismo: Fanfani e la politica estera italiana (1958-1963), cit., pp. 395-396.

57 «La destra interna al partito e anche la maggioranza dei "dorotei" entrarono in fibrillazione ai primi segni di ammorbidimento della rigorosa fedeltà italiana all'alleato d'oltreoceano, ritenendo l'atteggiamento di Fanfani del tutto strumentale, atto solo a compiacere l'alleato socialista. All'ambasciata americana fu riferito che Moro aveva addirittura minacciato le dimissioni da segretario della Dc per far rientrare la critica, definita una vera e propria 'rivolta della destra interna per l'annacquamento delle posizioni del governo su Cuba'. Ancor più duramente, Flaminio Piccoli [deputato democristiano esponente di spicco dei "dorotei"] andò a sostenere di fronte ai funzionari statunitensi che Fanfani era inaffidabile, perché dietro la vantata amicizia con Kennedy stavano le tendenze, sue e del suo gruppo, a volere un'Italia neutrale ed equidistante. Completava il quadro [...] il nervosismo crescente del nuovo presidente della Repubblica Segni, che più volte cercò di scavalcare il governo [...] per correggere a destra la politica estera del governo». FORMIGONI, Guido, Fanfani, la Dc e la ricerca di un nuovo discorso di politica estera, in GIOVAGNOLI, Agostino, TOSI, Luciano (a cura di), Amintore Fanfani e la politica estera italiana: atti del convegno di studi (Roma, 3-4 febbraio 2009), cit., pp. 88-89. 
«svuotamento dall'interno» dell'Alleanza ${ }^{5}$. Nel Psi emerse il travaglio dell'avvicinamento all'area governativa e del definitivo abbandono dell'alleanza con i comunisti. I socialisti autonomisti mostrarono, infatti, comprensione per l'orientamento dell'esecutivo e ribadirono che esso dimostrava che anche nella cornice della Nato c'era posto per iniziative di pace. I socialisti di sinistra si unirono invece al Pci nello stigmatizzare il moderatismo del governo59.

Concludendo il dibattito, Piccioni disse il 30 ottobre che il governo italiano si era sempre mostrato favorevole ai negoziati nel quadro Onu e che non aveva cessato di lavorare in tal senso. Affermò poi: «Il sottosegretario Russo ha trasmesso l'incoraggiamento dell'Italia all'azione conciliativa del segretario generale dell'Onu, U Thant; al tempo stesso abbiamo fatto conoscere alle parti più direttamente interessate nel conflitto il nostro desiderio di una soluzione pacifica. La solidarietà tra alleati atlantici si è rivelata totale» 60 .

Se dal punto di vista interno la crisi di Cuba rappresentò in Italia un momento di coesione tra le forze che promuovevano il centro-sinistra e di dissenso con i gruppi che, sia a destra che a sinistra, vi si opponevano, dal punto di vista delle relazioni internazionali costituì un momento di non minore importanza. Gli Stati Uniti, infatti, dopo lunghi mesi di progressivo avvicinamento e maturazione di una disposizione favorevole, si decisero ad appoggiare più apertamente l'esperimento dell'apertura ai socialisti, constatando che la coalizione che si veniva a formare in Italia aveva dimostrato di reggere alla prova dell'atlantismo ed aveva assicurato il totale sostegno alle decisioni della Casa Bianca ${ }^{61}$. Testimonianza di ciò risiede nel fatto che quando Fanfani, nel gennaio 1963, si recò negli Stati Uniti, fu accolto a Washington con grande

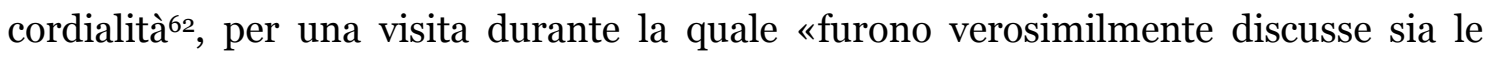

58 MARTELLI, Evelina, L'altro atlantismo: Fanfani e la politica estera italiana (1958-1963), cit., p. 395.

${ }_{9}$ Cfr. WOLLEMBORG, Leo, Stelle, strisce e tricolore. Trent'anni di vicende politiche fra Roma e Washington, cit., pp. 136-151.

6o MARTELLI, Evelina, L'altro atlantismo: Fanfani e la politica estera italiana (1958-1963), p. 395 .

${ }_{61}$ Cfr. NUTI, Leopoldo, Gli Stati Uniti e l'apertura a sinistra: importanza e limiti della presenza americana in Italia, cit., pp. 545-553.

62 «Il 16 gennaio 1963, durante il brindisi al termine di una colazione alla Casa Bianca che vedeva Fanfani ospite ufficiale in qualità di presidente del Consiglio, dopo averne elogiato i notevoli meriti nella recente straordinaria rinascita del Paese dopo il 1945, [Kennedy] ne tratteggiò in modo efficace e sintetico la concezione di base: Esso mi rammenta un aneddoto di Abramo Lincoln. Dopo che egli fu eletto presidente, qualcuno disse: Signor Lincoln, che farete con i vostri nemici? Lincoln rispose: Li distruggerò. Li farò miei amici. Questo sta facendo in Italia il primo ministro [chiosò Kennedy con chiaro riferimento all'apertura democristiana nei confronti dei socialisti]». GENTILONI SILVERI, Umberto, Fanfani visto da Washington, in GIOVAGNOLI, Agostino, TOSI, Luciano (a cura di), Amintore Fanfani e la politica estera italiana: atti del convegno di studi (Roma, 3-4 febbraio 2009), cit., p. 114. 
questioni riguardanti lo smantellamento dei Jupiter sia le scelte politiche che avrebbero successivamente governato l'Italia» 63 .

\section{Conclusioni}

Secondo Sergio Romano,

il centro-sinistra [...] divenne gradito a Washington nel momento in cui fu chiaro che la nuova costellazione [il governo Fanfani IV] [...] sarebbe stata ancora più infeudata all'America, per certi aspetti, del vecchio centrismo degasperiano. L'Italia era, infatti, la più piccola e la più debole delle grandi potenze, ma poteva sfruttare la forza degli altri e concorrere a risultati in cui il suo ruolo sarebbe stato valorizzato [...] Era, quella di Fanfani, la versione aggiornata e volontaristica di quella politica del peso determinante che il paese aveva praticato per gran parte della sua storia unitaria ${ }^{64}$.

Leopoldo Nuti, dal canto suo, ricorda che «in ogni discussione sulla politica estera di un futuro governo di centro-sinistra, la questione centrale era sempre la stessa: quale atteggiamento avrebbe assunto un governo di centro-sinistra nei confronti dell'Alleanza atlantica?»65. Le parole di questi due autorevoli studiosi dei rapporti italo-americani e più in generale delle relazioni di Roma con il resto del mondo, mettono in risalto ciò che si è cercato di evidenziare in questa ricerca, ovvero l'intima connessione esistente tra questioni domestiche italiane e politica estera. Si tratta di un dualismo che affonda le sue radici nella seconda metà del XIX secolo: Romano allude a un fil rouge che da Cavour giunge sino ad Amintore Fanfani per poi proseguire e arrivare ai giorni nostri. Il tradizionale "pendolarismo" italiano, fatto di machiavellismo e razionalità, costituisce, infatti, una costante della tradizione diplomatica del paese. Per dare credito a tale tesi si può partire addirittura dal 1855, quando Cavour e i dirigenti piemontesi vararono l'invio di truppe nella lontana Crimea allo scopo, raggiunto dati gli esiti successivi, di favorire il processo di unificazione nazionale. Ancora, si può pensare alla celebre enunciazione del più volte ministro degli Esteri Emilio Visconti-Venosta che, con fredda lucidità, amava sottolineare la necessità per gli italiani di essere «indipendenti sempre, isolati mai». Furono poi Sidney Sonnino e, soprattutto, Dino Grandi, vero

63 DI NOLFO, Ennio, La guerra fredda e l'Italia, cit., p. 431.

${ }^{64}$ ROMANO, Sergio, Lo scambio ineguale. Italia e Stati Uniti da Wilson a Clinton, Bari, Laterza, 1995, p. 94.

65 NUTI, Leopoldo, Gli Stati Uniti e l'apertura a sinistra: importanza e limiti della presenza americana in Italia, cit., p. 423. 
astro della politica del peso determinante, a cercare di fare dell'Italia l'ago della bilancia durante le due guerre mondiali.

Successivamente, nel secondo dopoguerra, il paese si trovò nella necessità di prendere posizione nell'ambito del bipolarismo emerso dopo gli eventi bellici. La scelta a "stelle e strisce" trovò il suo momento emblematico nel viaggio di De Gasperi a Washington del 1947. Da allora il primato dell'atlantismo nella politica estera italiana fu indubbio, nonostante le perplessità avanzate da diverse parti politiche, non solo della sinistra (si pensi a quei settori interni alla Dc che, sedotti dal neutralismo e dal non allineamento, osteggiarono l'ingresso nella $\mathrm{Nato}^{66}$ ). La testimonianza più rilevante dell'assoluta fedeltà agli Stati Uniti si deve probabilmente rintracciare proprio nel periodo considerato in questo lavoro. A cavallo fra la seconda metà degli anni Cinquanta e la formazione del primo governo di apertura al Psi, infatti, le questioni interne furono affrontate da Palazzo Chigi sempre con estrema cautela, attenzione volta a comprendere se le dinamiche italiane fossero gradite e approvate dalla Casa Bianca. In politica estera, inoltre, per Roma si dimostrò evidente che l'unica possibilità di concorrere alle scelte fondamentali dell'Alleanza e al suo dispositivo di difesa nucleare era quella di appoggiare le proposte statunitensi, orientate ad una maggiore integrazione ed al controllo americano sull'arsenale atomico della Nato. Per questo l'Italia, sotto la guida del secondo esecutivo Fanfani, accettò di ospitare sul suo territorio missili a media gittata provenienti da Washington e gestiti da un sistema che prevedeva il simultaneo consenso della Casa Bianca e di Roma per il loro utilizzo.

Durante la crisi di Cuba, Fanfani si strinse ancora di più attorno al blocco occidentale. Prima, il 23 ottobre, si rivolse a Kennedy con una missiva colma di parole piene di riguardo nei confronti del "grande alleato", che informò del suo pieno sostegno; poi non oppose alcuna resistenza alla rimozione dei Jupiter, che pure costituivano il simbolo tangibile dell'importanza che Washington conferiva al partner italiano, oltre che uno strumento concreto di difesa in caso di attacco sovietico. Quando gli Stati Uniti proposero il ritiro dei vettori, i dirigenti italiani accolsero la richiesta, non volendo contrastare gli interessi fondamentali dell'amministrazione americana. «Anche se consapevoli che il ritiro dei missili Jupiter era stato oggetto di trattativa segreta tra i sovietici e gli americani, mantennero un contegno riservato, accettando la versione

66 Nell'ambito della copiosa letteratura sul tema, cfr. FORMIGONI, Guido, La Democrazia cristiana e l'alleanza occidentale (1943-1953), Bologna, Il Mulino, 1996. 
ufficiale statunitense che il ritiro era dovuto all'obsolescenza di quel sistema difensivo» 67 .

Fanfani e il suo entourage compresero che un'eventuale azione di opposizione alle decisioni americane sarebbe stata tanto inutile quanto dannosa per il paese, che avrebbe rischiato di subire ritorsioni e di compromettere, di fatto, tutto il lavoro diplomatico che, da De Gasperi in avanti, gli statisti italiani avevano condotto allo scopo di ottenere la benevolenza di Washington. Per questo il governo di Roma decise intelligentemente di assecondare Kennedy che, in cambio, sostenne senza indugi il connubio Dc-Psi. La nuova coalizione di governo, in una situazione delicata come quella dell'ottobre 1962, aveva dimostrato di saper agire con compattezza e, soprattutto, nel pieno rispetto della fedeltà atlantica. Furono proprio gli sviluppi della crisi cubana a far comprendere definitivamente agli Stati Uniti come il centro-sinistra costituisse «una formula [...] decisamente migliore, dal punto di vista americano, di tutte le altre alternative disponibili» 68 .

${ }^{67}$ MARTELLI, Evelina, L'altro atlantismo: Fanfani e la politica estera italiana (1958-1963), cit., p. 445 .

${ }^{68}$ NUTI, Leopoldo, Gli Stati Uniti e l'apertura a sinistra: importanza e limiti della presenza americana in Italia, cit., p. 665. 


\section{* L'autore}

Matteo Anastasi ha conseguito la laurea in Storia presso l'Università Europea di Roma discutendo una tesi sull'attività del quarto governo di Amintore Fanfani. Attualmente si sta specializzando in Relazioni Internazionali presso l'Università LUISS Guido Carli, sotto la supervisione del professor Francesco Perfetti. I suoi campi di studio sono l'analisi del fenomeno politico dell' apertura a sinistra nel dopoguerra e la storia diplomatica italiana.

URL: < http://www.studistorici.com/progett/autori/\#Anastasi >

\section{Per citare questo articolo:}

ANASTASI, Matteo, «ll quarto governo Fanfani e la crisi di Cuba del 1962. Una chiave di volta per l'apertura a sinistra», Diacronie. Studi di Storia Contemporanea : II diritto miltante, 29/12/2014,

URL:< http://www.studistorici.com/2014/12/29/anastasi_numero_20/ >

Diacronie Studi di Storia Contemporanea 3 www.diacronie.it

Risorsa digitale indipendente a carattere storiografico. Uscita trimestrale.

redazione.diacronie@hotmail.it

Comitato di redazione: Jacopo Bassi - Luca Bufarale - Elisa Grandi - Deborah Paci - Fausto Pietrancosta - Matteo Tomasoni - Luca Zuccolo 